\title{
LINC01094/miR-577 axis regulates the progression of ovarian cancer
}

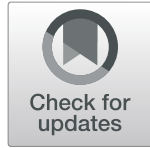

Jing $\mathrm{Xu}^{1}$, Ping Zhang ${ }^{2^{*}} \mathbb{D}$, Huajun Sun ${ }^{3}$ and Yang Liu ${ }^{4}$

\begin{abstract}
Background: Long intergenic non-coding RNA 01094 (LINC01094) is probably a novel regulator in cancer biology. This study aimed to probe into the function and mechanism of LINC01094 in ovarian cancer (OC).

Methods: Quantitative real-time polymerase chain reaction (qRT-PCR) assay was utilized to measure LINC01094 and miR577 expressions in OC tissues and cell lines. Western blot was used to examine the expressions of epithelial-mesenchymal transition (EMT)-related proteins, $\beta$-catenin, c-Myc and cyclin D1. Cell counting kit-8 (CCK-8) and Transwell assays were used to detect the proliferation, migration and invasion of SKOV3 and $3 \mathrm{AO}$ cells, respectively. Eventually, dual-luciferase reporter gene assay was employed to detect the regulatory relationship between miR-577 and LINC01094.
\end{abstract}

Results: LINC01094 expression was elevated in OC tissues and cell lines. High LINC01094 expression was associated with higher FIGO stage, lymph node metastasis and the shorter overall survival rate in patients with OC. Meanwhile, LINC01094 knockdown inhibited OC cell proliferation, migration, invasion and EMT. In addition, miR-577 was demonstrated to be a direct downstream target of LINC01094 in OC and inhibition of miR-577 reversed the biological effects of LINC01094 knockdown on OC cells. Additionally, LINC01094 / miR-577 axis regulated the expressions of $\beta$-catenin, c-Myc and cyclin D1 in OC cells.

Conclusion: LINC01094 promotes the proliferation, migration, invasion and EMT of OC cells by adsorbing miR-577.

Keywords: LINC01094, miR-577, Ovarian cancer, Cancer progression

\section{Introduction}

Ovarian cancer $(\mathrm{OC})$ is one of the deadliest cancers among women worldwide, triggering about 151,900 deaths per year $[1,2]$. Because of the lack of obvious symptoms at the early stage of the disease, most patients with OC have distant metastasis when being diagnosed, and the five-year survival rate is extremely low [3]. Therefore, it is of great significance to delve into the mechanism of OC tumorigenesis and progression and to find effective markers for early detection and therapeutic targets.

Long non-coding RNA (LncRNA) has been one of the research hotspots in recent years. Composed of more than 200 nucleotides in length, it is a kind of non-coding RNAs

\footnotetext{
*Correspondence: iamzhangping1978@163.com

2Department of Reproductive Medicine, Linyi People's Hospital, Fenghuang Street No. 233, Hedong District, Linyi, Shandong Province, China

Full list of author information is available at the end of the article
}

(ncRNAs) that cannot encode proteins $[4,5]$. Accumulating evidence shows that lncRNAs participate in a variety of biological activities [6]. Importantly, it is found that multiple lncRNAs are abnormally expressed in cancer cells, which modulate carcinogenesis and cancer progression [7]. Exploring the biological functions of IncRNA in the development of $\mathrm{OC}$ can provide insights for its diagnosis and treatment. A recent study reports that long intergenic noncoding RNA 01094 (LINC01094) is transcriptionally activated by FOXM1 and it promotes the progression of clear cell renal cell carcinoma [8]. However, in cancer biology, the role of LINC01094 has not been fully elucidated.

MicroRNA (miRNA) is a kind of ncRNA with 20-25 nucleotides. Similar to lncRNA, the abnormal expression of miRNAs is closely interrelated to the development of tumors and features prominently in biological activities, including cell differentiation, stress response, proliferation,

(c) The Author(s). 2020 Open Access This article is licensed under a Creative Commons Attribution 4.0 International License, which permits use, sharing, adaptation, distribution and reproduction in any medium or format, as long as you give appropriate credit to the original author(s) and the source, provide a link to the Creative Commons licence, and indicate if changes were made. The images or other third party material in this article are included in the article's Creative Commons licence, unless indicated otherwise in a credit line to the material. If material is not included in the article's Creative Commons licence and your intended use is not permitted by statutory regulation or exceeds the permitted use, you will need to obtain permission directly from the copyright holder. To view a copy of this licence, visit http://creativecommons.org/licenses/by/4.0/ The Creative Commons Public Domain Dedication waiver (http://creativecommons.org/publicdomain/zero/1.0/) applies to the data made available in this article, unless otherwise stated in a credit line to the data. 
apoptosis, migration, and so on [9]. Mounting evidence shows that miRNA can affect the growth and metastasis of OC cells [3, 10, 11]. Previous studies depict that miR-577 expression is abnormally reduced in diverse human malignancies, such as gastric cancer, breast cancer and glioma, suggesting that miR-577 is a tumor suppressor [12-14]. However, the expression and function of miR-577 in OC are not clear.

In this work, we proved that LINC01094 expression was elevated in OC and LINC01094 accelerated cell proliferation, migration, invasion and epithelial-mesenchymal transition (EMT) by targeting miR-577.

\section{Materials and methods}

\section{Clinical data and ethics statement}

Samples of OC tissues and adjacent non-tumor tissues were obtained from 93 patients in the surgery, whose diagnosis were confirmed by histopathology. All experiments were conducted according to the Declaration of Helsinki, and the study was endorsed by the Ethics Committee of the Qingdao Women's and Children's Hospital. Moreover, all patients enrolled signed the written informed consent.

\section{Cell culture}

The Institute of Biochemistry and Cell Biology, Chinese Academy of Sciences was the provider of human OC cell lines (SKOV3, HO8910, ES-2, HEY and 3AO cells) and immortalized human ovarian epithelial cell line MOODY. Cells were cultured in Dulbecco's Modified Eagle's Medium (DMEM, Gibco, Carlsbad, CA, USA) with 10\% fetal bovine serum (FBS; Hyclone, Logan, UT, USA), 100 $\mathrm{U} / \mathrm{ml}$ penicillin and $100 \mu \mathrm{g} / \mathrm{ml}$ streptomycin (Hyclone, Logan, UT, USA) at $37^{\circ} \mathrm{C}$ in $5 \% \mathrm{CO}_{2}$.

\section{Cell transfection}

Normal control siRNA (si-NC), LINC01094 siRNA, miR-577 mimics and miR-577 inhibitors were obtained from the Genepharma (Shanghai, China). Cell suspensions were prepared with trypsinized SKOV3 and $3 \mathrm{AO}$ cells. Then the cells were inoculated in a 6-well plate $\left(1 \times 10^{6}\right.$ cells/well $)$ and cultured. When reaching $80-90 \%$ confluence, the $\mathrm{OC}$ cells were incubated in fresh medium without serum and antibiotics for $3 \mathrm{~h}$. After that, transfection was performed with Lipofectamine 2000 (Invitrogen, Carlsbad, CA, USA). After $12 \mathrm{~h}$, the mixture of medium and lipofectamine was replaced by complete medium before the culture was continued for $24 \mathrm{~h}$. After that, the cells were harvested to detect the transfection efficiency and to conduct subsequently experiments.
Quantitative real-time polymerase chain reaction (qRTPCR) assay

Based on the manufacturer's instructions, TRIzol reagent (Takara, Dalian, China) was adopted to extract total RNA from tissues and cells. The cDNA was then synthesized using PrimeScript RT kit (TaKaRa, Dalian, China) and qRT-PCR assay was performed using SYBR Premix Ex Taq II (TaKaRa, Dalian, China) on ABI7500FAST RealTime PCR system (Applied Biosystems, Waltham, MA, UK), and the data were analyzed by Bio-Rad CFX96TM Manager (Bio-Rad, Hercules, CA, USA). Primers were designed, synthesized and provided by BGI (Shenzhen, China). The relative expressions of LINC01094 and miR577 were examined with the $2^{-\Delta \Delta C t}$ method.

\section{Cell counting kit-8 (CCK-8) assay}

CCK-8 kit (Dojindo, Kumamoto, Japan) was employed to evaluate the viability of transfected cells according to the manufacturer's protocols. Both SKOV3 and 3AO cells transfected with siRNAs were seeded in 96-well plates (1000 cells/well) with $100 \mu \mathrm{L}$ medium. The cell viability was detected at $0,24,48,72$, and $96 \mathrm{~h}$, respectively. At each time point, CCK-8 solution $(10 \mu \mathrm{L} /$ well $)$ was added, and the cells were cultured at $37^{\circ} \mathrm{C}$ in $5 \%$ $\mathrm{CO}_{2}$, and $2 \mathrm{~h}$ later, the absorbance of each well was measured employing a Model 680 Microplate Reader (BioRad, Richmond, CA, USA) at a wavelength of $450 \mathrm{~nm}$.

\section{Transwell assay}

In compliance with the manufacturer's protocol, Transwell chambers with $8 \mu \mathrm{m}$ pore size (Corning, NY, USA) were utilized to measure cell migration and invasion. Briefly, in cell migration experiments, SKOV3 and $3 \mathrm{AO}$ cells $\left(2 \times 10^{4}\right.$ cells $)$ were suspended in $200 \mu \mathrm{L}$ serum-free medium and placed into the upper chamber of each well, and $600 \mu \mathrm{L}$ medium containing 10\% FBS was dripped into the lower chamber. Cells were cultured at $37^{\circ} \mathrm{C}$ for $12 \mathrm{~h}$, and then the migrated cells were fixed with $4 \%$ paraformaldehyde and then stained with crystal violet solution. After that, the cells were counted under an inverted microscope. The procedures of invasion experiments were also performed as described above, except that the membrane of the Transwell chambers was coated by Matrigel (30 $\mu \mathrm{g} /$ well; BD, San Jose, CA, USA).

\section{Luciferase reporter gene assay}

The predicted LINC01094 sequence containing miR-577 binding site was amplified and inserted into empty luciferase reporter vector (Promega, Madison, WI, USA) to obtain LINC01094-wild type (WT) reporter vector. Meanwhile, the mutant (MUT) LINC01094 sequence was inserted into empty luciferase reporter vector to obtain LINC01094-MUT reporter vector. LINC01094-WT or LINC01094-MUT was co-transfected into HEK293T 
cells, respectively, with miR-577 mimic or control miRNA. Forty-eight hours later, relative luciferase activity of each group was measured using dual-luciferase reporter assay system (Promega, Madison, WI, USA).

\section{Western blot}

Total protein was extracted from SKOV3 and 3AO cells with RIPA buffer (Beyotime, Shanghai, China) supplemented with protease inhibitors. The same amount of protein in each group was separated by sodium dodecyl sulfate polyacrylamide gel electrophoresis and then the proteins were transferred to the nitrocellulose (NC) membrane (Pall Life Sciences, Port Washington, NY, USA). After that, the membrane was blocked with $5 \%$ skim milk for $1.5 \mathrm{~h}$ and then incubated with the corresponding specific primary antibody at $4{ }^{\circ} \mathrm{C}$ overnight. Then, the secondary antibody (1:5000) was employed to incubate the membrane at room temperature for $1 \mathrm{~h}$. Ultimately, electrochemical luminescence (ECL) kit (Beyotime, Shanghai, China) was used to detect the immunoreactivity. The primary antibodies used in this work included anti-E-cadherin antibody (Abcam, ab197751, 1:2000), anti-vimentin antibody (Abcam, ab92547, 1:1000), anti- $\beta$-catenin antibody (Abcam, ab16051, 1:1000), anti-c-Myc antibody (Abcam, ab32072, 1:2000), anti-cyclin D1 antibody (Abcam, ab16663, 1:2000) and anti-GAPDH antibody (Abcam, ab9484, 1:4000).

\section{Statistical processing}

Statistical analysis was conducted by SPSS22.0 statistical software (SPSS Inc., Chicago, IL, USA). All data were expressed as mean \pm standard deviation (SD). The significance of differences between the two groups was examined by $t$-test. GraphPad Prism 8.0 (GraphPad Software Inc., La Jolla, CA, USA) was utilized for drawing the figures. $P<0.05$ was statistically meaningful.

\section{Results}

LINC01094 expression was elevated in OC tissues and cell lines

First of all, Gene Expression Profiling Interactive Analysis (GEPIA) database (http://gepia.cancer-pku.cn/help. html) manifested that LINC01094 was differentially expressed in OC tissues and adjacent normal tissues, and LINC01094 expression in cancer tissues was remarkably higher than that in normal tissues (Fig. 1a). Consistently, qRT-PCR assay showed that the expression of LINC01094 in the collected OC samples was markedly higher in comparison with that in adjacent nontumor tissues (Fig. 1b). To explore the correlation between LINC01094 expression and clinical characteristics, we divided the OC samples into different groups based on FIGO stages and the status of lymphatic metastasis. As shown, the expression of LINC01094 in patients with higher FIGO stage and lymphatic metastasis was markedly higher than that in patients with lower stage and no lymphatic metastasis (Fig. 1c-d). These results demonstrated that LINC01094 was associated with the progression of OC. Importantly, GEPIA database also suggested that the overall survival rate of OC patients with high LINC01094 expression was lower than that in patients with low LINC01094 expression, indicating that LINC01094 had the potential to be a biomarker for evaluating the prognosis the OC patients (Fig. 1e). Next, qRT-PCR assay was conducted to determine the relative expression of LINC01094 in five OC cell lines and immortalized normal ovarian epithelial cell line MOODY. As shown, compared with in MOODY cells, LINC01094 expression in five OC cell lines was significantly up-regulated (Fig. 1f).

\section{Knockdown of LINC01094 inhibited the proliferation, migration, invasion and EMT of OC cells}

To pinpoint the function of LINC01094 on the malignant behaviors of OC cells, we selected SKOV3 and $3 \mathrm{AO}$ cell lines for further exploration. SK-3C and $3 \mathrm{AO}$ cells were transfected with si-LINC01094 or si-NC, and the knockdown efficiency was detected by qRT-PCR assay (Fig. 2a). CCK-8 assay results showed that compared to that in the si-NC group, the proliferation of SKOV3 and 3AO cells was significantly inhibited in the si-LINC01094 group (Fig. 2b). Transwell assay demonstrated that knocking down LINC01094 markedly reduced the migration and invasion of SKOV3 and $3 \mathrm{AO}$ cells (Fig. 2c-d). In addition, EMT is involved in the metastasis of cancer cells by endowing cancer cells with an invasive phenotype [15]. Therefore, we also explored the impact of LINC01094 on the EMT process. Western blot showed that knocking down LINC01094 could upregulate E-cadherin expression and down-regulate Vimentin expression, which illustrated that LINC01094 regulated the migration and invasion of $\mathrm{OC}$ cells partly by modulating EMT (Fig. 2e).

\section{MiR-577 was a direct downstream target of LINC01094}

To elaborate on the effect of LINC01094 in OC development, we used the bioinformatics tool StarBase 3.0 (http:// starbase.sysu.edu.cn) to predict the target miRNAs potentially regulated by LINC01094 and it was found that miR577 was a candidate target for LINC01094 (Fig. 3a). To validate this prediction, we determined the expression of miR-577 in OC tissues with qRT-PCR assay, and the results showed that miR-577 expression was remarkably declined in OC tissues (Fig. 3b). Dual-luciferase reporter assay showed that miR-577 mimic observably reduced the luciferase activity of LINC01094-WT reporter, but no significant change could be observed on mutant LINC01094-MUT reporter, indicating that LINC01094 could directly bind to miR-577 (Fig. 3c). In addition, 

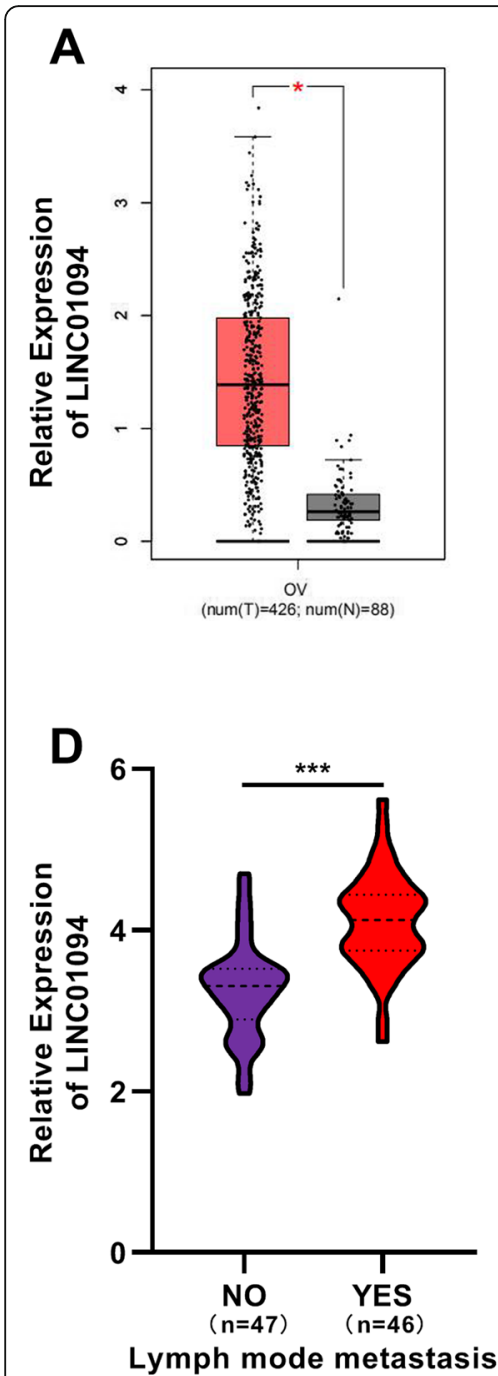
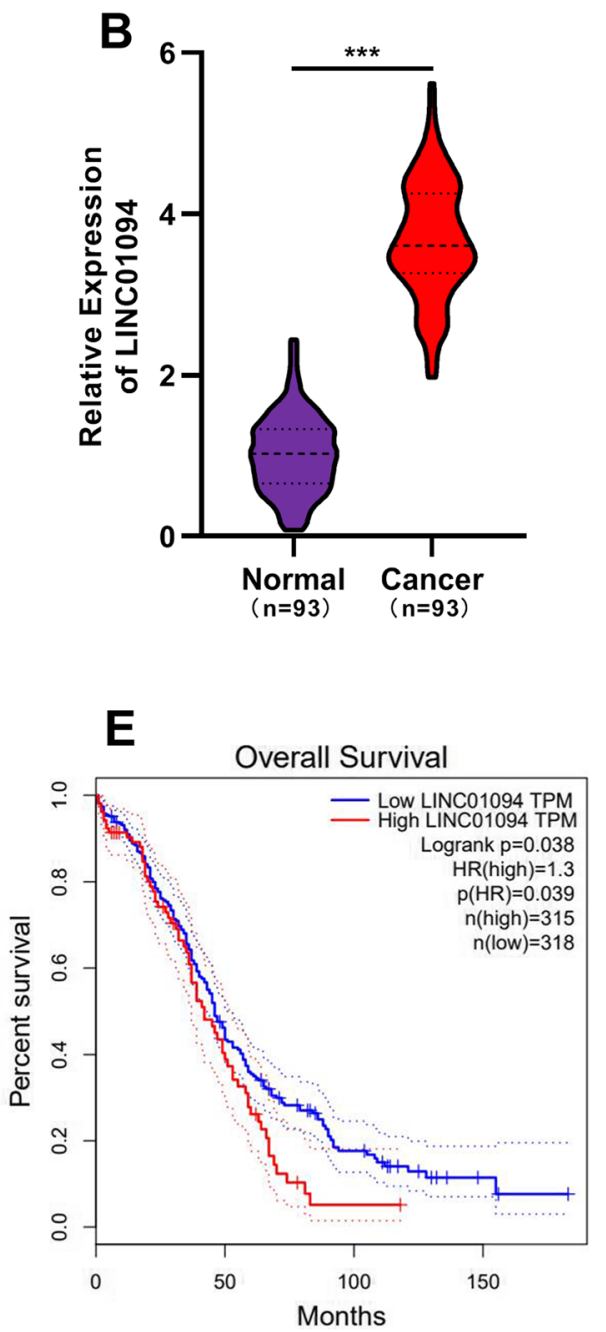
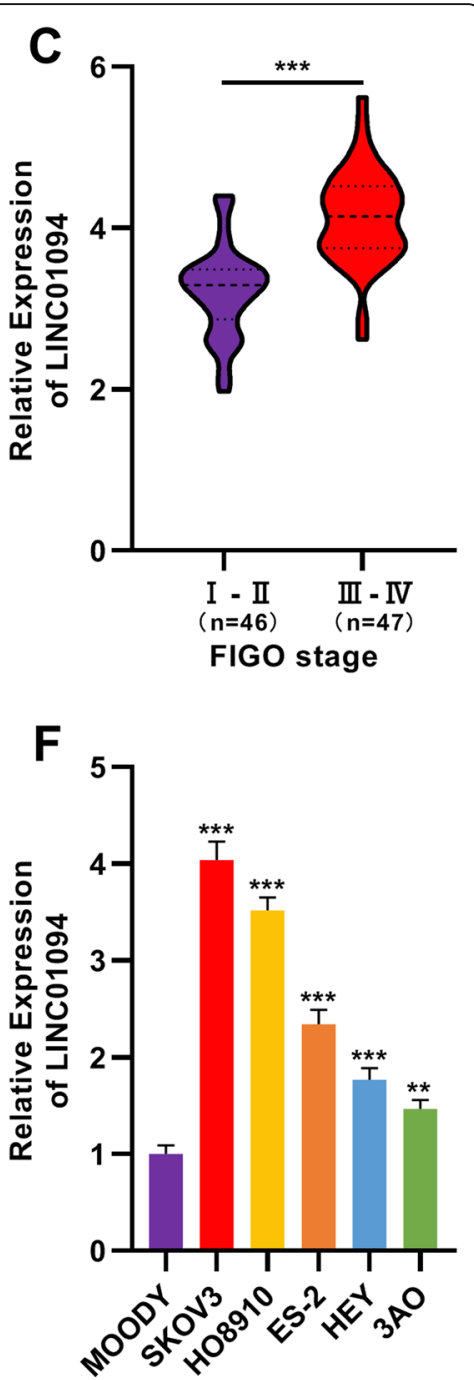

Fig. 1 LINC01094 expression was significantly up-regulated in OC tissues and cell lines. a Bioinformatics analysis based on GEPIA database showed the expressions of LINC01094 in OC tissues and adjacent normal tissues. $\mathbf{b}$ The expression of LINC01094 in OC tissues and adjacent tissues was detected by qRT-PCR assay. c-d The expression of LINC01094 was higher in patients with advanced FIGO stage (c) and lymph node metastasis (d). e GEPIA database was used to perform survival analysis of OC patients with high and low expression of LINC01094. $\mathbf{f}$ qRT-PCR assay showed that compared with in MOODY cells, the expression of LINC01094 in OC cell lines (SKOV3, HO8910, ES-2, HEY and 3AO cells) was all up-regulated. ${ }^{* *} P<0.01$ and ${ }^{* *} P<0.001$

LINC01094 knockdown dramatically enhanced miR-577 expression in SKOV3 and 3AO cells (Fig. 3d). Meanwhile, Pearson's correlation analysis showed that LINC01094 expression was negatively correlated with miR-577 expression in OC samples (Fig. 3e).

\section{Inhibition of miR-577 promoted the proliferation, migration and invasion of $O C$ cells}

To fathom the role of miR-577 in regulating the malignant behaviors of OC cells, miR-577 inhibitors or miRNA inhibitor control were transfected into SK-3C and $3 \mathrm{AO}$ cells, and the inhibition efficiency was detected by qRTPCR (Fig. 4a). CCK-8 assay manifested that compared with the control group, inhibition of miR-577 promoted the proliferation of SKOV3 and 3AO cells (Fig. 4b). Transwell analysis also showed that inhibition of miR-577 could significantly promote the migration and invasion of SKOV3 and 3AO cells (Fig. 4c-d). In addition, Western blot results showed that down-regulation of miR-577 expression could decrease the expression of E-cadherin and increase the expression of Vimentin (Fig. 4e). These results suggested that inhibition of miR-577 promoted the proliferation, migration and invasion of $\mathrm{OC}$ cells.

MiR-577 reversed the regulatory functions of LINC01094 on OC cell proliferation, migration and invasion

To investigate whether LINC01094 exerts biological functions through regulating miR-577, we performed a 


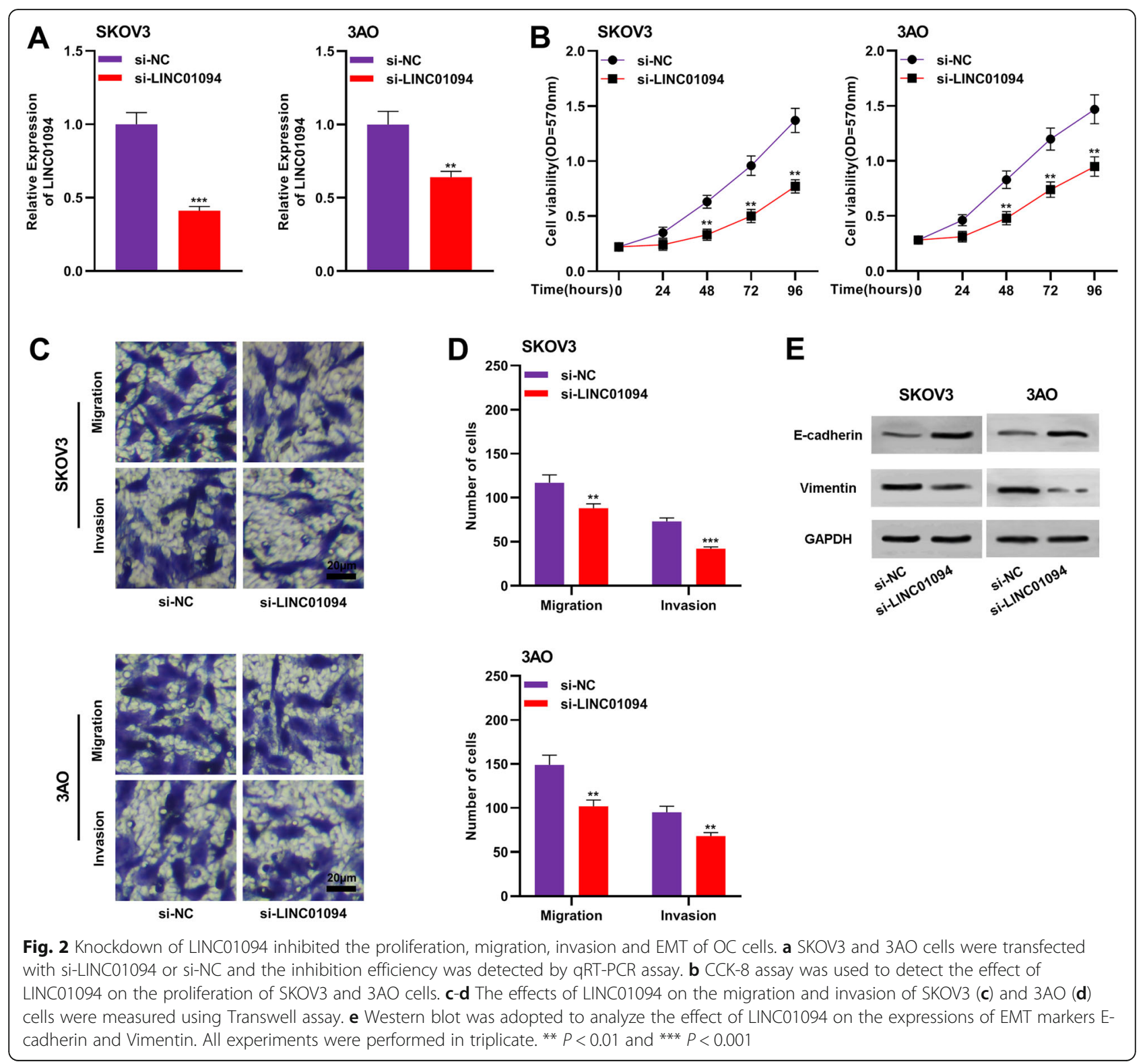

rescue experiment by inhibiting miR-577 expression in SKOV3 cells and 3AO cells with LINC01094 knockdown (Fig. 5a). CCK-8 assay verified that knockdown of LINC01094 inhibited the proliferation of SKOV3 cells and 3AO cells; miR-577 inhibition partially abolished this inhibitory effect (Fig. 5b). Subsequently, Transwell assay demonstrated that knocking down LINC01094 expression remarkably reduced the migration and invasion capacity of SKOV3 cells and 3AO cells; inhibiting miR577 partially reversed the decrease in the number of migrated and invaded cells (Fig. 5c-d). In addition, Western blot showed that knocking down LINC01094 expression could increase E-cadherin expression and reduce Vimentin expression in SKOV3 and 3AO cells; however, miR-
577 inhibitors partially offset the effect of LINC01094 on EMT process (Fig. 5e). Previous studies report that miR577 exerts its tumor-suppressive function via repressing Wnt/ $\beta$-catenin signaling [16-18], so we also detected whether LINC01094 could affect the activity of Wnt/ $\beta$ catenin signaling via regulating miR-577. Western blot was used to detect several Wnt/ $\beta$-catenin signaling related proteins. It was showed that LINC01094 knockdown reduced $\beta$-catenin, cyclin D1 and $\mathrm{c}-\mathrm{Myc}$ protein expressions in SKOV3 and 3AO cells, while miR-577 inhibitors partially reversed the inhibitory effect of knocking down LINC01094 on these proteins (Fig. 5f). These results indicated that LINC01094 could promote the proliferation, migration, invasion and EMT of OC cells 


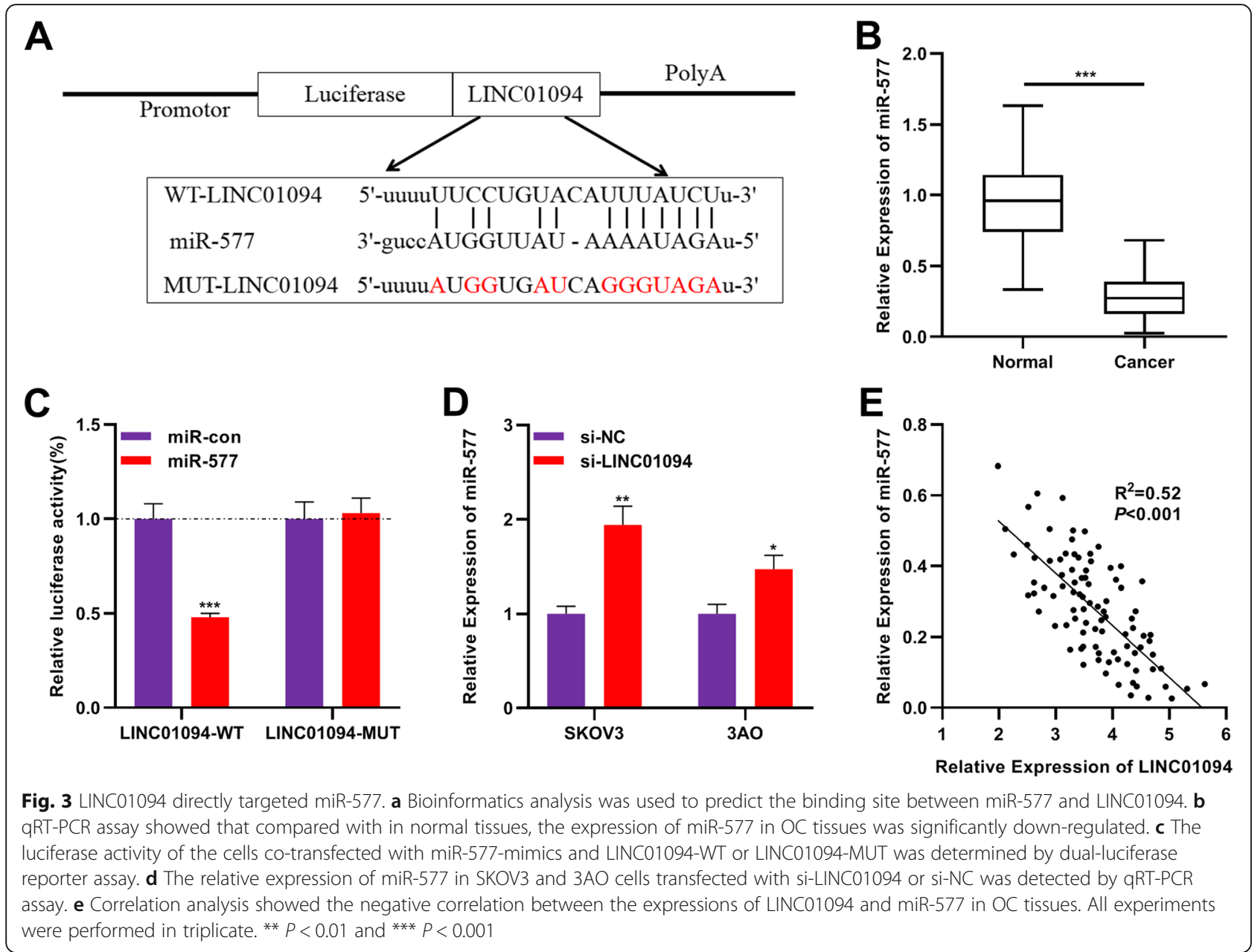

by repressing miR-577 expression and activating Wnt signaling.

\section{Discussion}

It is found that genetic and environmental factors can lead to the occurrence of OC [19]. However, due to the lack of obvious symptoms, effective screening and early diagnosis strategies, exceeding $70 \%$ of the patients are diagnosed with advanced $\mathrm{OC}$, resulting in poor prognosis and low survival rate [20]. LncRNA promotes the carcinogenesis, development and metastasis of many human malignancies [21]. Exploring the abnormal expression, function and mechanism of lncRNA in the development of OC can provide biomarkers and therapy targets [22]. For example, a recent study reports that a predictive model, based on the expressions of lncRNAs, GAS5, HCP5, PART1, SNHG11 and SNHG5 included, can identify OC patients at high risk of poor prognosis [23]; the up-regulation of LINC00319 expression accelerates the proliferation, migration and invasion of $\mathrm{OC}$ cells [24]; LncRNA PTAR expression is up-regulated in $\mathrm{OC}$ and it promotes the EMT and metastasis of OC cells
[25]. In this work, we focused on the role of LINC01094 in the progression of OC and found that LINC01094 was highly expressed in OC tissues and cell lines. Additionally, high LINC01094 expression was associated with advanced FIGO stage, lymph node metastasis and poor overall survival rate. Furthermore, in functional assays, we found that LINC01094 knockdown repressed the proliferation, migration, invasion and EMT of OC cells. The above results indicated that LINC01094 probably functioned as a tumor promoter in OC.

The various biological functions of lncRNA are largely dependent on its unique intracellular localization [26]. According to the competitive endogenous RNA (ceRNA) theory proposed in 2011, if the IncRNA is located in cytoplasma and contains miRNA response element (MRE), it probably functions as a molecular sponge to competitively decoy miRNAs, thus modulating the translation of mRNAs $[27,28]$. In cancer biology, this mechanism is proved to play a crucial role. For instance, LINC00319 regulates the expression of NACC1 by targeting miR-423-5p to promote the progression of OC [24]; IncRNA HOXD-AS1 facilitates the proliferation and invasion of $\mathrm{OC}$ cells by targeting miR- 


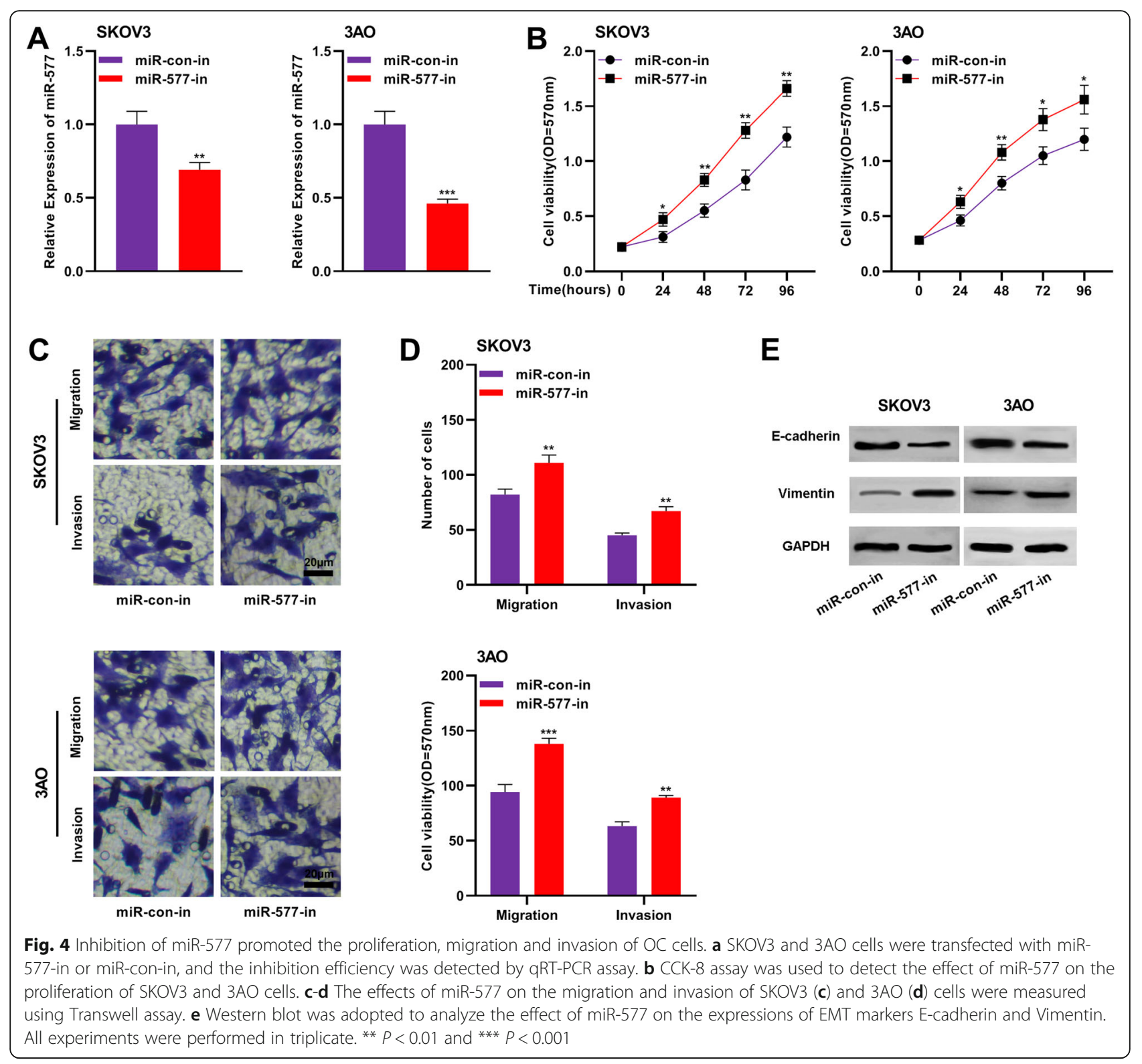

133-3p and activating Wnt / $\beta$-catenin signaling pathway [29]. LINC01094 is proved to be a ceRNA for miR-224-5p in clear cell renal cell carcinoma, and it increases the translation of oncogene CHSY1 via competitively binding to miR224-5p [8]. In the present study, we confirmed that miR-577 was another direct downstream target of LINC01094. It was demonstrated that inhibition of miR-577 promoted the malignant biological behaviors of OC cells. It was also observed that knockdown of LINC01094 increased the expression of miR-577, and LINC01094 expression was negatively correlated with miR-577 expression in OC samples. We also found that the biological functions of LINC01094 was partly dependent on its regulatory function on miR-577. Previous studies demonstrate that miR-577 is a well-recognized tumor suppressor and it negatively regulates a lot of oncogenes and oncogenic pathways, including Sphk2, Smurf1, Rab14, Rab25, LRP6, $\beta$-catenin, Wnt2b, and so on [12-14, 16-18, 30-32]. It is possible that LINC01094 can indirectly up-regulate these oncogenes expressions to promote OC progression via repressing miR-577.

The Wnt/ $\beta$-catenin signaling pathway consists of a series of proteins, which is encoded by proto-oncogenes and tumor suppressors. The interrelationships among these proteins are vital in regulating the process of cell proliferation, apoptosis, differentiation, migration, adhesion, and so on [33]. Activation of the Wnt leads to the stabilization of $\beta$-catenin and its accumulation in cytoplasm; $\beta$-catenin is then transferred into the nucleus and modulates the transcription of genes [34]. Meanwhile, many oncogenes are transcriptionally activated by Wnt 


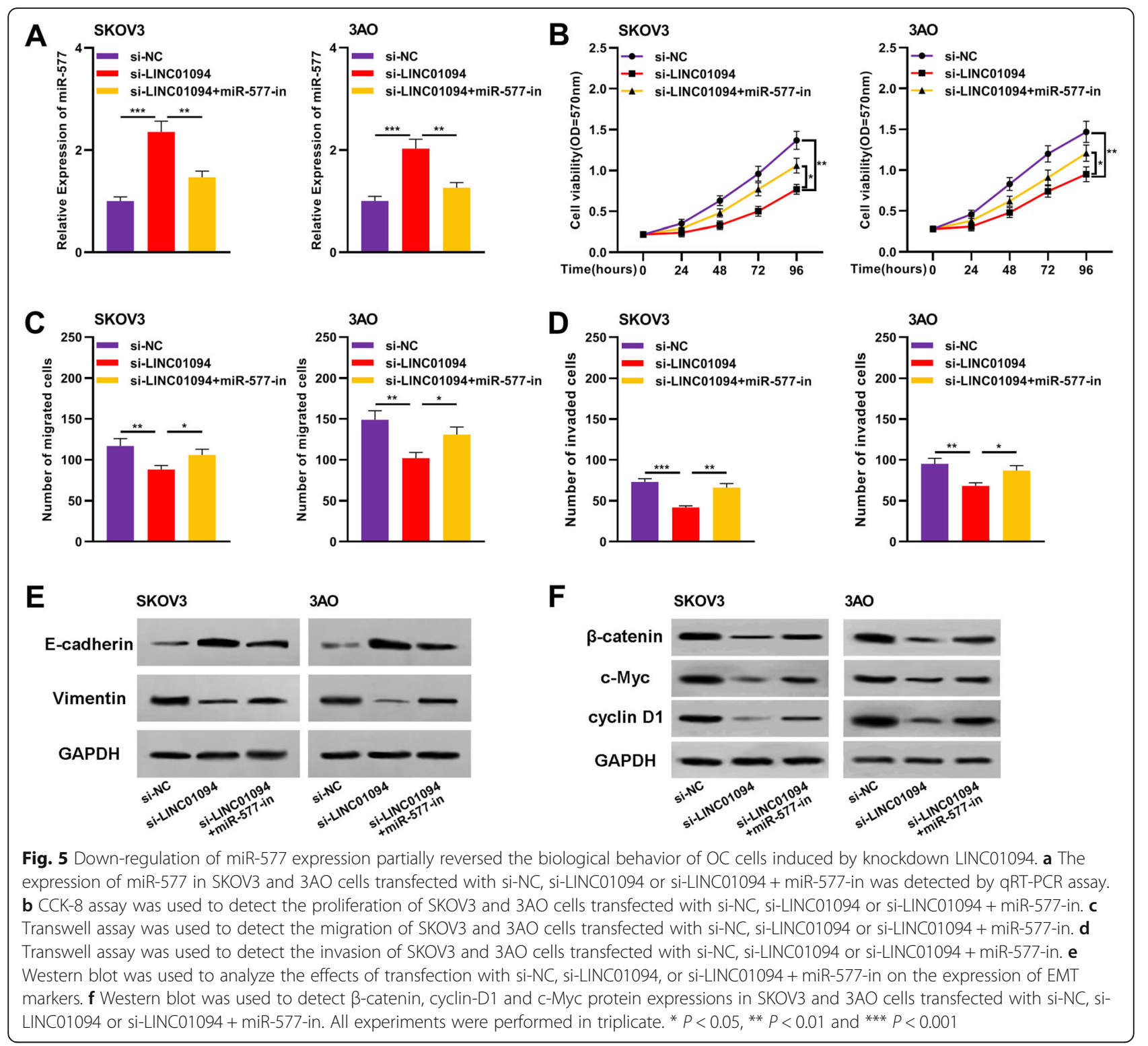

signaling cascades, such as c-myc and cyclin D1 [35]. In OC, Wnt/B-catenin signaling also functions importantly. For instance, PYGB promotes OC cell migration and invasion via modulating the Wnt signaling pathway [36]. By activating Wnt signaling, STAT3 promotes the stemness and spheroid formation of OC cells [37]. Interestingly, many targets of miR-577 are pivotal components of $\beta$-catenin/ Wnt signaling such as LRP6, $\beta$-catenin and Wnt2b, which suggests that miR-577 is a crucial modulator in $\beta$-catenin/ Wnt signaling. The present work demonstrated that the effects of knocking down LINC01094 on Wnt signaling pathway in OC cells could also be partially reversed by the down-regulation of miR-577. Therefore, these data suggested that LINC01094 activated Wnt signaling pathway through adsorbing miR-577, and promoted the proliferation, migration, invasion and EMT process of OC cells.
In short, in this work, we prove that LINC01094 expression is increased in OC and LINC01094 promotes cell proliferation, migration, invasion and EMT by targeting miR-577 and activating $\mathrm{Wnt} / \beta$-catenin signaling. This research provides new ideas for OC gene therapy. However, animal experiments are needed to further validate our demonstrations in the following studies. Additionally, other downstream miRNAs of LINC01094 remain to be screened and verified.

\section{Acknowledgments}

Not applicable.

\section{Authors' contributions}

$\mathrm{Xu}$ Jing and Zhang Ping developed the original design and analyzed the data. Sun Huajun and Liu Yang performed the initial literature review, wrote the first draft of the report and prepared the manuscript. All authors read and approved the final manuscript. 


\section{Funding}

Not applicable.

\section{Availability of data and materials \\ Not applicable.}

\section{Ethics approval and consent to participate}

Not applicable.

\section{Consent for publication}

Not applicable.

\section{Competing interests}

The authors declare that they have no competing interests.

\section{Author details}

'Department of Obstetrics and Gynecology, Zibo Hospital of Integrated Traditional Chinese and Western Medicine, Zibo, Shandong Province, China. ${ }^{2}$ Department of Reproductive Medicine, Linyi People's Hospital, Fenghuang Street No. 233, Hedong District, Linyi, Shandong Province, China. ${ }^{3}$ Department of Obstetrics and Gynecology, Yantai Yeda Hospital, Yantai, Shandong Province, China. ${ }^{4}$ Department of Gynecology, Qingdao Women's and Children's Hospital, Qingdao, Shandong Province, China.

Received: 5 May 2020 Accepted: 17 September 2020

Published online: 17 October 2020

\section{References}

1. Yu KH, Hu V, Wang F, et al. Deciphering serous ovarian carcinoma histopathology and platinum response by convolutional neural networks. BMC Med. 2020:18(1):236

2. Torre LA, Trabert B, DeSantis CE, et al. Ovarian cancer statistics, 2018. CA Cancer J Clin. 2018;68(4):284-96.

3. Xiao F, Xiao S, Xue M. miR-139 controls viability of ovarian Cancer cells through apoptosis induction and exosome shedding inhibition by targeting ATP7A. Onco Targets Ther. 2019;12:10727-37.

4. Lu S, Jiang X, Su Z, Cui Z, Fu W, Tai S. The role of the long non-coding RNA HOXA11-AS in promoting proliferation and metastasis of malignant tumors. Cell Biol Int. 2018 Dec:42(12):1596-601.

5. Gourvest M, Brousset P, Bousquet M. Long Noncoding RNAs in Acute Myeloid Leukemia: Functional Characterization and Clinical Relevance. Cancers (Basel). 2019;11(11):1638.

6. Wu X, Yuan Y, Ma R, Xu B, Zhang R. IncRNA SNHG7 affects malignant tumor behaviors through downregulation of EZH2 in uveal melanoma cell lines. Oncol Lett. 2020;19(2):1505-15.

7. Qiu C, Li S, Sun D, Yang S. IncRNA PVT1 accelerates progression of nonsmall cell lung cancer via targeting miRNA-526b/EZH2 regulatory loop. Oncol Lett. 2020:19(2):1267-72.

8. Jiang Y, Zhang H, Li W, Yan Y, Yao X, Gu W. FOXM1-activated LINC01094 promotes clear cell renal cell carcinoma development via MicroRNA 224-5p/ CHSY1. Mol Cell Biol. 2020;40(3):e00357-19.

9. Rupaimoole R, Slack FJ. MicroRNA therapeutics: towards a new era for the management of cancer and other diseases. Nat Rev Drug Discov. 2017;16(3): 203-22.

10. Wang Y, Lei $X$, Gao C, et al. MiR-506-3p suppresses the proliferation of ovarian cancer cells by negatively regulating the expression of MTMR6. J Biosci. 2019:44(6):126.

11. He L, Zhu W, Chen Q, et al. Ovarian cancer cell-secreted exosomal miR-205 promotes metastasis by inducing angiogenesis. Theranostics. 2019:9(26): 8206-20.

12. Luo Y, Wu J, Wu Q, et al. miR-577 regulates TGF- $\beta$ induced Cancer progression through a SDPR-modulated positive-feedback loop with ERKNF-KB in gastric Cancer. Mol Ther. 2019;27(6):1166-82.

13. Yin C, Mou Q, Pan X, Zhang G, Li H, Sun Y. MiR-577 suppresses epithelialmesenchymal transition and metastasis of breast cancer by targeting Rab25. Thorac Cancer. 2018:9(4):472-9.

14. Wei $\mathrm{N}$, Wei H, Zhang H. Long non-coding RNA ZEB1-AS1 promotes glioma cell proliferation, migration and invasion through regulating miR-577. Eur Rev Med Pharmacol Sci. 2018;22(10):3085-93.

15. Gladilin $\mathrm{E}$, Ohse $\mathrm{S}$, Boerries $\mathrm{M}$, et al. TGFß-induced cytoskeletal remodeling mediates elevation of cell stiffness and invasiveness in NSCLC. Sci Rep. 2019;9(1):7667.
16. Wang B, Sun L, Li J, Jiang R. miR-577 suppresses cell proliferation and epithelial-mesenchymal transition by regulating the WNT2B mediated WNT/ $\beta$-catenin pathway in non-small cell lung cancer. Mol Med Rep. 2018;18(3): 2753-61.

17. Jiang Z, Jiang C, Fang J. Up-regulated Inc-SNHG1 contributes to osteosarcoma progression through sequestration of miR-577 and activation of WNT2B/WNT/B-catenin pathway. Biochem Biophys Res Commun. 2018; 495(1):238-45.

18. Zhang W, Shen C, Li C, et al. miR-577 inhibits glioblastoma tumor growth via the Wnt signaling pathway. Mol Carcinog. 2016;55(5):575-85.

19. Fortner RT, Poole EM, Wentzensen NA, et al. Ovarian cancer risk factors by tumor aggressiveness: an analysis from the ovarian Cancer cohort consortium. Int J Cancer. 2019 Jul 1;145(1):58-69.

20. Moore K, Colombo N, Scambia G, et al. Maintenance Olaparib in patients with newly diagnosed advanced ovarian Cancer. N Engl J Med. 2018; 379(26):2495-505

21. Mathy NW, Chen XM. Long non-coding RNAs (IncRNAs) and their transcriptional control of inflammatory responses. J Biol Chem. 2017;292(30): 12375-82.

22. Do H, Kim W. Roles of oncogenic long non-coding RNAs in Cancer development. Genomics Inform. 2018;16(4):e18.

23. Zhao Q, Fan C. A novel risk score system for assessment of ovarian cancer based on co-expression network analysis and expression level of five IncRNAs. BMC Med Genet. 2019:20(1):103.

24. Du W, Feng Z, Sun Q. LncRNA LINC00319 accelerates ovarian cancer progression through miR-423-5p/NACC1 pathway. Biochem Biophys Res Commun. 2018:507(1-4):198-202.

25. Liang $\mathrm{H}$, Yu T, Han Y, et al. LncRNA PTAR promotes EMT and invasionmetastasis in serous ovarian cancer by competitively binding miR-101-3p to regulate ZEB1 expression. Mol Cancer. 2018;17(1):119.

26. Kopp F, Mendell JT. Functional classification and experimental dissection of long noncoding RNAs. Cell. 2018;172(3):393-407.

27. Sardina DS, Alaimo S, Ferro A, Pulvirenti A, Giugno R. A novel computationa method for inferring competing endogenous interactions. Brief Bioinform. 2017:18(6):1071-81.

28. Liu Y, Wang Y, Fu X, Lu Z. Long non-coding RNA NEAT1 promoted ovarian cancer cells' metastasis through regulation of miR-382-3p/ROCK1 axial. Cancer Sci. 2018;109(7):2188-98.

29. Zhang Y, Dun Y, Zhou S, Huang XH. LncRNA HOXD-AS1 promotes epithelial ovarian cancer cells proliferation and invasion by targeting miR-133a-3p and activating Wnt/ß-catenin signaling pathway. Biomed Pharmacother. 2017;96: $1216-21$

30. Sun Y, Shi T, Ma Y, Qin H, Li K. Long noncoding RNA LINC00520 accelerates progression of papillary thyroid carcinoma by serving as a competing endogenous RNA of microRNA-577 to increase Sphk2 expression. Cell Cycle. 2020;19(7):787-800

31. Li T, Xing Y, Yang F, et al. LncRNA SNHG3 sponges miR-577 to up-regulate SMURF1 expression in prostate cancer. Cancer Med. 2020;9(11):3852-62.

32. Yang J, Liang B, Hou S. TMPO-AS1 promotes cervical cancer progression by upregulating RAB14 via sponging miR-577. J Gene Med. 2019;21(11):e3125.

33. Yuan C, Gou X, Deng J, Dong Z, Ye P, Hu Z. FAK and BMP-9 synergistically trigger osteogenic differentiation and bone formation of adipose derived stem cells through enhancing Wnt- $\beta$-catenin signaling. Biomed Pharmacother. 2018;105:753-7.

34. Zhu X, Yuan C, Tian C, et al. The plant sesquiterpene lactone parthenolide inhibits Wnt/ $\beta$-catenin signaling by blocking synthesis of the transcriptional regulators TCF4/LEF1. J Biol Chem. 2018:293(14):5335-44.

35. Vallée A, Guillevin $R$, Vallée $J N$. Vasculogenesis and angiogenesis initiation under normoxic conditions through Wnt/B-catenin pathway in gliomas. Rev Neurosci. 2018;29(1):71-91.

36. Zhou Y, Jin Z, Wang C. Glycogen phosphorylase B promotes ovarian cancer progression via Wnt/ $\beta$-catenin signaling and is regulated by miR-133a-3p. Biomed Pharmacother. 2019;120:109449.

37. Chen MW, Yang ST, Chien MH, et al. The STAT3-miRNA-92-Wnt signaling pathway regulates spheroid formation and malignant progression in ovarian Cancer. Cancer Res. 2017:77(8):1955-67.

\section{Publisher's Note}

Springer Nature remains neutral with regard to jurisdictional claims in published maps and institutional affiliations. 